\title{
Positive Self-adjoint Operator Extensions with Applications to Differential Operators
}

\author{
B. M. Brown, W. D. Evans and I. G. Wood®
}

\begin{abstract}
In this paper we consider extensions of positive operators. We study the connections between the von Neumann theory of extensions and characterisations of positive extensions via decompositions of the domain of the associated form. We apply the results to elliptic second order differential operators and look in particular at examples of the Laplacian on a disc and the Aharonov-Bohm operator.
\end{abstract}

Mathematics Subject Classification. Primary 47A20; Secondary 35J15, 47A07, 47B25, 47F05.

Keywords. Operator extensions, Von Neumann theory, Sesquilinear form, Elliptic operators, Aharonov-Bohm operator.

\section{Introduction}

Let $A$ be a closed strictly positive symmetric operator with dense domain $D(A)$ and range $R(A)$ in a Hilbert space $H$. In [11,12], Krein proved that there is a one to one correspondence between the set of positive self-adjoint extensions $A_{B}$ of $A$ and a set of pairs $\left\{N_{B}, B\right\}$, where $N_{B}$ is a subspace of the kernel $N$ of $A^{*}$ and $B$ is a positive self-adjoint operator with domain and range in $N_{B}$. Krein's result was subsequently developed further by Visik [15] and Birman [3]; this work of the three authors will be referred to as the KVB theory. An important extension of the KVB theory was made in [8] to a pair of closed densely defined operators $A, A^{\prime}$, which form a dual pair in the sense that $A \subset\left(A^{\prime}\right)^{*}$ and are such that $A \subset A_{\beta} \subset\left(A^{\prime}\right)^{*}$ for an operator $A_{\beta}$ with a bounded inverse. The results in [8] include those of KVB when $A=A^{\prime}$. Of particular interest to us in [8] is the application of the abstract theory to the case when $A$ is generated by an elliptic differential expression acting in a bounded smooth domain $\Omega$ in $\mathbb{R}^{n}$. In this case the self-adjoint extensions of $A$ are determined by boundary conditions on the boundary $\partial \Omega$ of $\Omega$.

The Authors are grateful to the anonymous referees for providing useful comments that helped improve the paper. 
In [5], results in Rellich [13], Kalf [9] and Rosenberger [14] were applied to the KVB theory to determine all the positive self-adjoint extensions of a positive Sturm-Liouville operator with minimal conditions on the coefficients. Our objective in this paper is to investigate what can be achieved by applying similar methods to two problems on bounded domains in $\mathbb{R}^{n}, n \geq 2$; in the first $A$ is generated by a second order elliptic expression, and in the second it is the Aharonov-Bohm operator on a punctured disc. Our analysis depends on an abstract result which incorporates the von Neumann theory concerning all the self-adjoint extensions of any symmetric operator.

Denote by $A_{F}, a_{F}[\cdot, \cdot]$ the Friedrichs extension and associated sesquilinear form of $A$. Then for all $u \in D\left(A_{F}\right)$ and $v \in Q\left(A_{F}\right)$ we have

$$
a_{F}[u, v]=\left(A_{F} u, v\right),
$$

where $(\cdot, \cdot)$ is the inner product of $H$, and $D\left(A_{F}\right)$ is dense as a subspace of $Q\left(A_{F}\right)$ with inner product $a_{F}[\cdot, \cdot]$ (see $[7$, Chapter IV] for more on the relation between sesquilinear forms, operators and their Friedrichs extension). By the KVB theory, $\hat{A}$ is a positive self-adjoint extension of $A$ if and only if, $\hat{A}=A_{B}$, where $B$ is a positive self-adjoint operator acting in a subspace $N_{B}$ of $N$ and $A_{B}, B$ have associated forms $a_{B}, b$, respectively which satisfy

$$
a_{B}=a_{F}+b, \quad Q\left(A_{B}\right)=Q\left(A_{F}\right) \dot{+} Q(B) .
$$

Thus any $u \in Q\left(A_{B}\right)$ can be uniquely written as $u=u_{F}+u_{N}$, where $u_{F} \in Q\left(A_{F}\right), u_{N} \in Q(B)$. There are two distinguished positive self-adjoint extensions of $A$, namely the Friedrichs (or strong) extension $A_{F}$ and the Krein-von Neumann (or weak) extension $A_{K}$. These are extremal in the sense that any positive self-adjoint extension $\hat{A}$ of $A$ satisfies $A_{K} \leq \hat{A} \leq A_{F}$ in the form sense. In (1.1), the Krein-von Neumann extension $A_{K}$ corresponds to $B=0, N_{B}=N$, and the Friedrichs extension $A_{F}$ to $B=\infty, Q(B)=0$, that is, $B$ acts trivially on a zero dimensional space.

\section{Positive Extensions and the Von Neumann Theory}

The von Neumann theory characterises the self-adjoint extensions of any closed densely defined symmetric operator $T$. Denoting the deficiency spaces $\operatorname{ker}\left(T^{*} \mp i I\right)$ by $N_{ \pm}$, we have

$$
D\left(T^{*}\right)=D(T) \dot{+} N_{+} \dot{+} N_{-},
$$

and $T_{S}$ is a self-adjoint extension of $T$ if and only if there is a unitary operator $U\left(T_{S}\right): N_{+} \rightarrow N_{-}$such that

$$
D\left(T_{S}\right)=D(T) \dot{+}\left(I+U\left(T_{S}\right)\right) N_{+} .
$$

Let $u, v \in D\left(T^{*}\right)$. Then by the von Neumann theory, there exist unique $u_{0}, v_{0} \in D(T)$ and $u_{ \pm}, v_{ \pm} \in N_{ \pm}$such that $u=u_{0}+u_{+}+u_{-}$and $v=$ $v_{0}+v_{+}+v_{-}$. 
It follows that

$$
\begin{aligned}
\left(T^{*} u, v\right)-\left(u, T^{*} v\right)= & \left(T u_{0}+i u_{+}-i u_{-}, v_{0}+v_{+}+v_{-}\right) \\
& -\left(u_{0}+u_{+}+u_{-}, T v_{0}+i v_{+}-i v_{-}\right) \\
= & \left(T u_{0}, v_{+}+v_{-}\right)+i\left(u_{+}-u_{-}, v_{0}+v_{+}+v_{-}\right) \\
& +i\left(u_{0}+u_{+}+u_{-}, v_{+}-v_{-}\right)-\left(u_{+}+u_{-}, T v_{0}\right) \\
= & -i\left(u_{0}, v_{+}-v_{-}\right)+i\left(u_{+}-u_{-}, v_{0}+v_{+}+v_{-}\right) \\
& +i\left(u_{0}+u_{+}+u_{-}, v_{+}-v_{-}\right)-i\left(u_{+}-u_{-}, v_{0}\right) \\
= & 2 i\left[\left(u_{+}, v_{+}\right)_{N_{+}}-\left(u_{-}, v_{-}\right)_{N_{-}}\right] .
\end{aligned}
$$

Let $P_{+}$and $P_{-}$denote the projections from $D\left(T^{*}\right)$ to $N_{+}$and $N_{-}$with respect to the decomposition (2.1) and let $U: N_{+} \rightarrow N_{-}$be unitary. Set $\tilde{\Lambda}_{0}=U P_{+}+P_{-}$and $\tilde{\Lambda}_{1}=-i U P_{+}+i P_{-}$. Then, for any $u, v \in D\left(T^{*}\right)$

$$
\left(T^{*} u, v\right)-\left(u, T^{*} v\right)=\left(\tilde{\Lambda}_{0} u, \tilde{\Lambda}_{1} v\right)-\left(\tilde{\Lambda}_{1} u, \tilde{\Lambda}_{0} v\right)
$$

(see [10, Theorem 3]). The triple $\left(N_{+}, \tilde{\Lambda}_{0}, \tilde{\Lambda}_{1}\right)$ is a boundary triple (also known as a space of boundary values) for $T$.

Given a self-adjoint extension $T_{S}$ of $T$, we now choose

$$
\begin{aligned}
& \Lambda_{0}\left(T_{S}\right)=U\left(T_{S}\right) P_{+}+P_{-}, \\
& \Lambda_{1}\left(T_{S}\right)=-i U\left(T_{S}\right) P_{+}+i P_{-} .
\end{aligned}
$$

Then, from $(2.2), \operatorname{ker} \Lambda_{1}\left(T_{S}\right)=\mathcal{D}\left(T_{S}\right)$ and we obtain, for all $u, v \in D\left(T^{*}\right)$

$$
\left(T^{*} u, v\right)-\left(u, T^{*} v\right)=\left(\Lambda_{0}\left(T_{S}\right) u, \Lambda_{1}\left(T_{S}\right) v\right)-\left(\Lambda_{1}\left(T_{S}\right) u, \Lambda_{0}\left(T_{S}\right) v\right) .
$$

Let $T=A$ be positive and $B$ a positive self-adjoint operator on a subspace $N_{B}$ of the kernel of $A^{*}$ with domain $D(B)$. By [2, Theorem 3.1], the domain of the self-adjoint extension $A_{B}$ of $A$ corresponding to $B$ is

$$
\mathcal{D}\left(A_{B}\right)=\left\{u_{0}+A_{F}^{-1}(B v+f)+v: u_{0} \in \mathcal{D}(A), v \in \mathcal{D}(B), f \in N \cap \mathcal{D}(B)^{\perp}\right\} .
$$

Remark 2.1. The special case $B=0, N_{B}=N$ gives the domain of the Kreinvon Neumann extension $A_{K}$, namely

$$
\mathcal{D}\left(A_{K}\right)=\mathcal{D}(A)+N,
$$

the sum being a direct sum since $A$ is strictly positive. It follows that

$$
\operatorname{ker}\left(A_{K}\right)=N \text {. }
$$

The Friedrichs extension is characterised by the choice of $B$ as acting trivially on $N_{B}=\{0\}$. Following the approach of [2], we can set $b[u]=\infty$ for $u \in N \backslash Q(B)$. It follows from (1.1) that $Q\left(A_{B}\right)=Q\left(A_{F}\right)$ if and only if $Q(B)=\{0\}$. Since $A_{F}$ is the only self-adjoint extension of $A$ with domain in $Q\left(A_{F}\right)$ it follows that its domain is determined by $b[u]=\infty$ for all $u \in N \backslash\{0\}$.

Theorem 2.2. Let $A_{B}$ be a positive self-adjoint extension of the positive operator $A$ associated with the pair $\left\{B, N_{B}\right\}$. Let $u \in D\left(A_{B}\right)$, where $u=u_{F}+w$, $u_{F}=u_{0}+A_{F}^{-1}(B w+v), u_{0} \in \mathcal{D}(A), w \in \mathcal{D}(B), v \in N \cap \mathcal{D}(B)^{\perp}$. Then

$$
b[w, \zeta]=\left(\Lambda_{0}\left(A_{B}\right) u, \Lambda_{1}\left(A_{B}\right) \zeta\right), \quad \forall \zeta \in Q(B),
$$


where $\Lambda_{0}\left(A_{B}\right)=U\left(A_{B}\right) P_{+}+i P_{-}$and $\Lambda_{1}\left(A_{B}\right)=-i U\left(A_{B}\right) P_{+}+i P_{-}$.

Proof. Let $\varphi=\theta+\zeta \in Q\left(A_{B}\right)$ with $\theta \in Q\left(A_{F}\right)$ and $\zeta \in Q(B)$. Then on the one hand, we have

$$
\left(A_{B} u, \varphi\right)=\left(A^{*} u, \varphi\right)=\left(A^{*} u_{F}, \varphi\right)
$$

since $w \in N$, and on the other hand,

$$
\begin{aligned}
\left(A_{B} u, \varphi\right) & =a_{B}[u, \varphi]=a_{F}\left[u_{F}, \theta\right]+b[w, \zeta] \\
& =\left(A_{F} u_{F}, \theta\right)+b[w, \zeta] \\
& =\left(A^{*} u_{F}, \theta\right)+b[w, \zeta] .
\end{aligned}
$$

On combining (2.11) and (2.12) we get

$$
b[w, \zeta]=\left(A^{*} u_{F}, \varphi-\theta\right)=\left(A^{*} u_{F}, \zeta\right)=\left(A^{*} u, \zeta\right),
$$

and as $A^{*} \zeta=0$, Eq. (2.6) yields

$$
b[w, \zeta]=\left(\Lambda_{0}\left(A_{B}\right) u, \Lambda_{1}\left(A_{B}\right) \zeta\right)-\left(\Lambda_{1}\left(A_{B}\right) u, \Lambda_{0}\left(A_{B}\right) \zeta\right) .
$$

Since $\operatorname{ker} \Lambda_{1}\left(A_{B}\right)=D\left(A_{B}\right)$, (2.10) follows.

Let $\left\{\psi_{k}\right\}$ be an orthonormal-basis of $Q(B)$, where $B$ is a positive selfadjoint operator in $N_{B} \subset N$, and let $w=\sum_{j} w_{j} \psi_{j}, \zeta=\sum \zeta_{k} \psi_{k}$ and $b_{j k}=$ $b\left[\psi_{j}, \psi_{k}\right]$. Then $b[w, \zeta]=\sum_{j, k} b_{j k} w_{j} \overline{\zeta_{k}}$ and from (2.10) and the fact that $\operatorname{ker} \Lambda_{1}\left(A_{B}\right)=D\left(A_{B}\right), u=u_{F}+w \in D\left(A_{B}\right)$ if and only if

$$
\forall k .\left(\Lambda_{0}\left(A_{B}\right) u, \Lambda_{1}\left(A_{B}\right) \psi_{k}\right)=\sum_{j} b_{j k} w_{j} \overline{\zeta_{k}} .
$$

\section{Elliptic Differential Operators of Second Order}

In this section we shall apply the above abstract theory to the case when $A$ is the closure of a symmetric second-order differential operator in $L^{2}(\Omega)$ defined by

$$
A^{\prime} u:=(-\nabla \cdot p \nabla+q) u=\left(-\sum_{i, j=1}^{n} D_{i} p_{i j} D_{j}+q\right) u, \quad u \in C_{0}^{\infty}(\Omega),
$$

subject to conditions on the coefficients $p_{i j}, q$ and the domain $\Omega$. The assumptions are the ones made in [1] which weaken the smoothness requirements on the coefficients and the boundary of $\Omega$ made by Grubb [8]. In the following definition of a boundary regularity class, $B_{p, q}^{s}$ is the Besov space of order $s$ (see $\left[1\right.$, Section 2]), and we set $x=\left(x^{\prime}, x_{n}\right), x^{\prime} \in \mathbb{R}^{n-1}, x_{n} \in \mathbb{R}$.

Definition 3.1. The boundary $\partial \Omega$ is said to be of class $B_{p, q}^{M-\frac{1}{2}}$ if for each $x \in \partial \Omega$ there exist an open neighbourhood $U$ satisfying the following: for a suitable choice of coordinates on $\mathbb{R}^{n}$, there is a function $\gamma \in B_{p, q}^{M-\frac{1}{2}}\left(\mathbb{R}^{n-1}\right)$ such that $U \cap \Omega=U \cap \mathbb{R}_{\gamma}^{n}$ and $U \cap \partial \Omega=U \cap \partial \mathbb{R}_{\gamma}^{n}$, where $\mathbb{R}_{\gamma}^{n}=\left\{x \in \mathbb{R}^{n}: x_{n}>\right.$ $\left.\gamma\left(x^{\prime}\right)\right\}$. 
In the list of assumptions to be made, we shall denote the boundary of $\Omega$ by $\Sigma$, and $H_{t}^{s}$ is a Bessel potential space (a Sobolev space for $s \in \mathbb{N}$ ), which we write as $H^{s}$ when $t=2$; see $\left[1\right.$, Section 2] for definitions of $H_{t}^{s}(\Omega)$ and $H_{t}^{s}(\Sigma)$.

\section{Assumptions}

1. There exists $c_{0}>0$ such that for all $x \in \Omega$ and $\xi \in \mathbb{R}^{n}$

$$
\sum_{i, j=1}^{n} p_{i j}(x) \xi_{i} \xi_{j} d x \geq c_{0}\|\xi\|^{2} .
$$

2. There exists $c>0$ such that

$$
\|u\|_{1}^{2}=\int_{\Omega}\left(p|\nabla u|^{2}+q|u|^{2}\right) d x \geq c\|u\|^{2}, \quad u \in C_{0}^{\infty}(\Omega) .
$$

The completion of $C_{0}^{\infty}(\Omega)$ with respect to the norm $\|\cdot\|_{1}$ is the form domain $Q\left(A_{F}\right)$ of $A$.

3. The boundary $\Sigma$ is of class $B_{r, 2}^{\frac{3}{2}}$ and the coefficients $p$ and $q$ of $A$ lie in $H_{t}^{1}(\Omega)$ and $L_{t}(\Omega)$, respectively, under the constraints $n \geq 2,2<r<\infty$, $2<t \leq \infty$, and

$$
1-\frac{n}{t} \geq \frac{1}{2}-\frac{n-1}{r}>0 .
$$

Remark 3.2. Our third assumption is Assumption 2.18 in [1]. Therefore, we have that for $v \in Q\left(A_{F}\right), \gamma_{0} v=0$, where $\gamma_{0}$ is the trace operator which maps $v$ into its value on $\Sigma$ (see [1, Theorem 2.11]). Moreover, in the notation of $[1,6]$, denote the solution of

$$
A w=0 \text { in } \Omega, w=u \text { on } \Sigma .
$$

by

$$
w=K_{\gamma}^{0} \gamma_{0} u .
$$

Then by [1, Theorem 5.4], for all $s \in[0,2]$,

$$
K_{\gamma}^{0}: H^{s-1 / 2}(\Sigma) \rightarrow H^{s}(\Omega)
$$

is continuous,

$$
K_{\gamma}^{0}: H^{s-1 / 2}(\Sigma) \rightarrow Z_{0}^{s}(A):=\left\{u \in H^{s}(\Omega): A u=0\right\}
$$

is a homeomorphism, and

$$
\gamma_{0}: Z_{0}^{s}(A) \rightarrow H^{s-1 / 2}(\Sigma)=\left(K_{\gamma}^{0}\right)^{-1} .
$$

We remark that under the more restrictive assumptions that $\Omega$ is a bounded domain whose boundary is an $(n-1)$-dimensional $C^{\infty}$ manifold, and the coefficients $p_{j k}, q$ of $A^{\prime}$ in (3.1) lie in $C^{\infty}(\bar{\Omega})$ these properties were already shown by Grubb in [8].

Theorem 3.3. Let the above assumptions hold and let $A_{B}$ be a positive selfadjoint extension of $A$. For $u \in D\left(A_{B}\right)$, we have $u=u_{F}+w$ for some $u_{F} \in D\left(A_{F}\right), w \in Q(B)$, and for all $\zeta \in Q(B)$

$$
b[w, \zeta]=\int_{\Omega}(-\nabla p \nabla+q) u_{F} \bar{\zeta} d x
$$


If $\left\{\psi_{k}\right\}$ is an orthonormal basis of $Q(B)$ then, with $b_{j k}$ as in (2.14),

$$
\forall k . \sum_{j} b_{j k} w_{j}+\int_{\Omega}(\nabla p \nabla-q) u_{F} \overline{\psi_{k}} d x=0 .
$$

Proof. Let $a_{B}[\cdot, \cdot], a_{F}[\cdot, \cdot], b[\cdot, \cdot]$ denote the forms associated with $A_{B}, A_{F}, B$, respectively. For $u, \varphi \in Q\left(A_{B}\right)$ we have the decompositions

$$
\begin{aligned}
& u=u_{F}+w, \quad\left(u_{F} \in Q\left(A_{F}\right), w=K_{\gamma}^{0} \gamma_{0} u \in Q(B)\right), \\
& \varphi=\varphi_{F}+\zeta, \quad\left(\varphi_{F} \in Q\left(A_{F}\right), \zeta=K_{\gamma}^{0} \gamma_{0} \varphi \in Q(B)\right) .
\end{aligned}
$$

If $u \in D\left(A_{B}\right)$, it has the decomposition $u=A_{F}^{-1} A^{*} u+\left(u-A_{F}^{-1} A^{*} u\right)$, i.e., $u_{F}=A_{F}^{-1} A^{*} u$ and $w=u-A_{F}^{-1} A^{*} u$, since $u_{F} \in D\left(A^{*}\right) \cap Q\left(A_{F}\right)=D\left(A_{F}\right)$ and $w \in Q\left(A_{B}\right) \cap N=Q(B)$. Now, let $\varphi=\varphi_{F}+\zeta \in Q\left(A_{B}\right)$. Then

$$
\left(A_{B} u, \varphi\right)=\int_{\Omega}(-\nabla p \nabla+q) u \bar{\varphi} d x
$$

and furthermore,

$$
\begin{aligned}
\left(A_{B} u, \varphi\right) & =(u, \varphi)_{1}=a_{B}[u, \varphi]=a_{F}\left[u_{F}, \varphi_{F}\right]+b[w, \zeta] \\
& =\int_{\Omega}(-\nabla p \nabla+q) u_{F} \overline{\varphi_{F}} d x+b[w, \zeta] \\
& =\int_{\Omega}(-\nabla p \nabla+q) u \overline{\varphi_{F}} d x+b[w, \zeta] .
\end{aligned}
$$

Combining (3.11) and (3.12), we get

$$
b[w, \zeta]=\int_{\Omega}(-\nabla p \nabla+q) u\left(\bar{\varphi}-\overline{\varphi_{F}}\right) d x=\int_{\Omega}(-\nabla p \nabla+q) u_{F} \bar{\zeta} d x .
$$

Now let $\left\{\psi_{k}\right\}$ be an orthonormal-basis of $Q(B), w=\sum w_{j} \psi_{j}$ and $\zeta=$ $\sum \zeta_{k} \psi_{k}$. Then

$$
b[w, \zeta]=\sum_{j, k} b_{j k} w_{j} \overline{\zeta_{k}}
$$

while the right-hand side of (3.13) is

$$
\sum_{k} \int_{\Omega}(-\nabla p \nabla+q) u \overline{\zeta_{k} \psi_{k}} d x
$$

Consequently

$$
\sum_{k} \overline{\zeta_{k}}\left[\sum_{j} b_{j k} w_{j}+\int_{\Omega}(\nabla p \nabla-q) u \overline{\psi_{k}} d x\right]=0
$$

and equivalently,

$$
\sum_{k} \overline{\zeta_{k}}\left[\sum_{j} b_{j k} w_{j}+\int_{\Omega}(\nabla p \nabla-q) u_{F} \overline{\psi_{k}} d x\right]=0 .
$$


As $\left\{\zeta_{k}\right\}$ is an arbitrary sequence in $\ell^{2}$, the 'boundary condition' associated with $A_{B}$ is given by

$$
\forall k . \sum_{j} b_{j k} w_{j}+\int_{\Omega}(\nabla p \nabla-q) u_{F} \overline{\psi_{k}}=0 .
$$

Corollary 3.4. The boundary condition associated with $A_{B}$ is given by

$$
\forall k \cdot \sum_{j} b_{j k} w_{j}=\left(\nu_{1} u_{F}, \gamma_{0} \psi_{k}\right)
$$

where $\nu_{1} u=\sum_{j, k=1}^{n} n_{j} \gamma_{0}\left[p_{j k} D_{k} u\right]$ and $\mathbf{n}=\left(n_{1}, \ldots, n_{n}\right)$ is the interior unit normal.

Proof. Since $u, \psi_{k} \in D\left(A^{*}\right)$, we can use [1, Theorem 6.1] to write

$$
\begin{aligned}
& \int_{\Omega}(\nabla p \nabla-q) u_{F} \overline{\psi_{k}} d x-\int_{\Omega} u_{F}(\nabla p \nabla-q) \overline{\psi_{k}} d x \\
& \quad=\left(\gamma_{0} u_{F}, \Gamma_{1} \psi_{k}\right)_{\Sigma}-\left(\Gamma_{1} u_{F}, \gamma_{0} \psi_{k}\right)_{\Sigma},
\end{aligned}
$$

where $\Gamma_{1}$ is the "regularised" Neumann operator given by $\Gamma_{1} u=\nu_{1} u-$ $P_{\gamma_{0}, \nu_{1}} \gamma_{0} u$ and $P_{\gamma_{0}, \nu_{1}} \gamma_{0}$ is the Dirichlet-to-Neumann map $P_{\gamma_{0}, \nu_{1}} \gamma_{0}=\nu_{1} K_{\gamma}^{0}$. Under the smoothness conditions assumed, the trace maps $\gamma_{0}, \Gamma_{1}, \operatorname{map} D\left(A^{*}\right)$ continuously into $H^{-1 / 2}(\Sigma), H^{1 / 2}(\Sigma)$, respectively. The terms on the righthand side of (3.16) therefore represent in fact, $H^{-1 / 2}, H^{1 / 2}$-duality products over the boundary $\Sigma$, which are extensions of the $L^{2}(\Sigma)$ inner products (see [1, Theorem 6.1]).

Since $(\nabla p \nabla-q) \psi_{k}=0$ and $u_{F} \in Q\left(A_{F}\right)$, two of the four terms in (3.16) vanish and, as $\Gamma_{1} u_{F}=\nu_{1} u_{F}$, we get that the boundary condition in (3.14) becomes

$$
\forall k \cdot \sum_{j} b_{j k} w_{j}=\left(\nu_{1} u_{F}, \gamma_{0} \psi_{k}\right)_{\Sigma}
$$

Remark 3.5. The Friedrichs extension is determined by the boundary condition $\gamma_{0} u=0$. Under the additional smoothness assumptions on $\Omega$ and the coefficients of $A^{\prime}$ in (3.1) in [8], the Friedrichs extension has domain $H_{0}^{1}(\Omega) \cap H^{2}(\Omega)$.

Remark 3.6. The Krein-von Neumann extension corresponds to $B=0, Q_{B}=$ $N_{B}=N=\operatorname{ker}\left(A^{*}\right)$ and so

$$
Q\left(A_{B}\right)=Q\left(A_{F}\right)+N, \quad a_{B}[u]=a_{F}\left[u_{F}\right],
$$

when $u=u_{F}+w, u_{F} \in Q\left(A_{F}\right), w \in N$. Thus in (3.15), $b_{j k}=0$ for all $j, k$ and $\nu_{1} u_{F}=\Gamma_{1} u_{F}$. Since $\nu_{1}$ maps $D\left(A^{*}\right)$ continuously into $H^{-1 / 2}(\Sigma)$ and $\gamma_{0}$ is a homeomorphism of $N$ onto $H^{1 / 2}(\Sigma)$, it follows from (3.15) that the boundary condition satisfied by the Krein-von Neumann extension is

$$
\nu_{1} u_{F}=0 .
$$


Since $w=K_{\gamma}^{0} \gamma_{0} u$ we have

$$
\nu_{1} u_{F}=\left(\nu_{1}-\nu_{1} K_{\gamma}^{0} \gamma_{0}\right) u=\Gamma_{1} u .
$$

Remark 3.7. On combining (2.10) and (3.17) we have

$$
\left(\Lambda_{0}\left(A_{B}\right) u, \Lambda_{1}\left(A_{B}\right) \psi_{k}\right)=\left(\nu_{1} u_{F}, \gamma_{0} \psi_{k}\right)_{\Sigma}
$$

For the Krein-von Neumann extension $\Lambda_{K} \psi_{k}=0$, so we again get

$$
\Gamma_{1} u=\nu_{1} u_{F}=0
$$

as the Krein-von Neumann boundary condition.

Example 3.8. We consider extensions of the positive operator $A=-\Delta+1$ when $\Omega$ is the unit disc in $\mathbb{R}^{2}$. According to (3.15), $v=v_{F}+w$ lies in the domain of an extension $A_{B}$ if and only if

$$
\left(\nu_{1} v_{F}, \gamma_{0} \psi_{k}\right)_{\Sigma}=\sum_{j} b_{j k}\left(w, \psi_{j}\right)
$$

for all $k$, where $\left\{\psi_{k}\right\}$ is an orthonormal-basis of the subspace $Q(B)$ in $N=$ $\operatorname{ker} A^{*}$.

Let $-\Delta \psi+\psi=0$ and put $\psi(r, \theta)=R(r) \Theta(\theta)$, where $x=(r, \theta)$ are polar co-ordinates. Then since

$$
\Delta \psi=R^{\prime \prime} \Theta+\frac{1}{r} R^{\prime} \Theta+\frac{1}{r^{2}} R \Theta^{\prime \prime}=R \Theta=\psi,
$$

we get

$$
r^{2} \frac{R^{\prime \prime}}{R}+r \frac{R^{\prime}}{R}-r^{2}=n^{2}
$$

and $\frac{\Theta^{\prime \prime}}{\Theta}=-n^{2}$ with constant $n$ and $\Theta(0)=\Theta(2 \pi)$; thus $\Theta_{n}(\theta)=e^{i n \theta}, n \in \mathbb{Z}$ and we seek the $L^{2}(0,1 ; r d r)$ solutions of

$$
r^{2} R^{\prime \prime}+r R^{\prime}-r^{2} R=n^{2} R, \quad r \in[0,1]
$$

These solutions are given by the modified Bessel functions $I_{n}(r)$ and $K_{n}(r)$.

For $n \geq 1, K_{n}(r)$ does not lie in $L^{2}(0,1 ; r d r)$. The function $K_{0}(r)$ has a logarithmic singularity at 0 , which means that $\Delta K_{0}$ is not zero in the sense of distributions, excluding $K_{0}$ from $N$. Therefore

$$
\psi_{k}(r, \theta)=I_{k}(r) e^{i k \theta}, \quad k \in \mathbb{Z}
$$

is a basis for $N$; note that $I_{-k}=I_{k}$.

For $k \in \mathbb{Z}$

$$
\gamma_{0} \psi_{k}(\theta)=\psi_{k}(1, \theta)=I_{k}(1) e^{i k \theta}
$$

and since $v_{F} \in D\left(A_{F}\right)$, we have $\nu_{1} v_{F}=\frac{\partial v_{F}}{\partial \nu}$. On expanding $v_{F}$ in $\theta$ in terms of its Fourier series,

$$
v_{F}(r, \theta)=\sum_{n \in \mathbb{Z}} v_{F, n}(r) e^{i n \theta}
$$

we derive

$$
\left.\frac{\partial v_{F}}{\partial \nu}\right|_{\partial \Omega}=\sum_{n \in \mathbb{Z}} \frac{\partial v_{F, n}}{\partial r}(1) e^{i n \theta} .
$$


Consequently $v=v_{F}+w \in D\left(A_{B}\right)$ if and only if for all $k \in \mathbb{Z}$

$$
\begin{aligned}
\sum_{j} b_{j k}\left(w, \psi_{j}\right) & =\left(\nu_{1} v_{F}, \gamma_{0} \psi_{k}\right)_{\Sigma} \\
& =\int_{0}^{2 \pi} \sum_{n \in \mathbb{Z}} \frac{\partial v_{F, n}}{\partial r}(1) e^{i n \theta} I_{k}(1) e^{-i k \theta} d \theta \\
& =2 \pi \frac{\partial v_{F, k}}{\partial r}(1) I_{k}(1) .
\end{aligned}
$$

Remark 3.9. 1. For the Krein-von Neumann extension, $v=v_{F}+w \in$ $D\left(A_{K}\right)$ if and only if for all $k \in \mathbb{Z}$ we have

$$
0=2 \pi \frac{\partial v_{F, k}}{\partial r}(1) I_{k}(1)
$$

As $I_{k}(1) \neq 0$ for all $k \in \mathbb{Z}$, this implies that

$$
v_{F}(1, \theta)=\frac{\partial v_{F}}{\partial r}(1, \theta)=0
$$

and hence $v_{F} \in D(A)$. As there are no restrictions on $w$, we get $D\left(A_{K}\right)=$ $D(A)+N$, as expected. Also, the boundary condition satisfied by any $u \in D\left(A_{K}\right)$ is $\Gamma_{1} u=0$, where $\Gamma_{1}=\nu_{1}-P_{\gamma_{0}, \nu_{1}} \gamma_{0}$ is the regularised Neumann operator.

2. For the Friedrichs extension, we formally have $b_{j k}=\infty$ for all $j, k$ in (3.19). This implies that $w$ must be orthogonal to all the $\psi_{k}$. As $w \in N$, this gives $w=0$.

\section{Aharonov-Bohm Operator}

Let $\Omega=\{x:|x|<1\} \backslash\{0\} \subset \mathbb{R}^{2}$, and let $A$ be the closure in $L^{2}(\Omega)$ of $A^{\prime} 1_{C_{0}^{\infty}(\Omega)}$, where

$$
A^{\prime}:=-(\nabla+i M)^{2} .
$$

Here, the Aharonov-Bohm magnetic potential

$$
M:=\alpha \frac{1}{\left(x_{1}^{2}+x_{2}^{2}\right)}\left(-x_{2}, x_{1}\right)=\alpha \frac{e_{\theta}}{r}, \quad \alpha \in(0,1),
$$

where $x=(r \cos \theta, r \sin \theta)$ in polar co-ordinates and $e_{\theta}=(-\sin \theta, \cos \theta)$ is the unit vector orthogonal to $e_{r}=x / r$. Then

$$
\operatorname{curl} M=0 \text { in } \Omega \text {, and } M \cdot e_{r}=0 .
$$

For $u \in C_{0}^{\infty}(\Omega)$ we have

$$
\begin{aligned}
\left(A^{\prime} u, u\right) & =\int_{\Omega}|(\nabla+i M) u|^{2} d x \\
& =\int_{0}^{1} \int_{0}^{2 \pi}\left(\left|\frac{\partial u}{\partial r}\right|^{2}+r^{-2}\left|i \frac{\partial u}{\partial \theta}+\alpha u\right|^{2}\right) r d r d \theta .
\end{aligned}
$$


The sequence $\left\{\varphi_{k}(\theta): k \in \mathbb{Z}\right\}$, where $\varphi_{k}(\theta)=\frac{e^{-i k \theta}}{\sqrt{2 \pi}}$, is an orthonormal basis for $L^{2}(0,2 \pi)$ and hence any $u \in L^{2}(\Omega)$ has the representation

$$
u(r, \theta)=\Sigma_{k} u_{k}(r) \varphi_{k}(\theta),
$$

where

$$
u_{k}(r)=\int_{0}^{2 \pi} u(r, \theta) \overline{\varphi_{k}(\theta)} d \theta .
$$

On substituting in (4.3), we have, with $\lambda_{k}=k+\alpha$

$$
\left(A^{\prime} u, u\right)=\sum_{k} \int_{0}^{1}\left(\left|u_{k}^{\prime}(r)\right|^{2}+\frac{\lambda_{k}^{2}}{r^{2}}\left|u_{k}(r)\right|^{2}\right) r d r .
$$

Since $\min \left\{\left|\lambda_{k}\right| / r: k \in \mathbb{Z}, 0<r<1\right\} \geq \min \{\alpha, 1-\alpha\}>0$, it follows that $A$ is strictly positive and its form domain $Q\left(A_{F}\right)$ is the completion of $C_{0}^{\infty}(\Omega)$ with respect to the norm given by the square root of

$$
a_{F}[u]:=\sum_{k} \int_{0}^{1}\left(\left|u_{k}^{\prime}(r)\right|^{2}+\frac{\lambda_{k}^{2}}{r^{2}}\left|u_{k}(r)\right|^{2}\right) r d r .
$$

Let $B$ be a positive self-adjoint operator acting in a closed subspace $N_{B}$ of $N=\operatorname{ker} A^{*}$ which is associated with the self-adjoint extension $A_{B}$ of $A$ in the KVB theory, and let $a_{B}[\cdot, \cdot], a_{F}[\cdot, \cdot], b[\cdot, \cdot]$ be the forms of $A_{B}, A_{F}, B$, respectively.

For $u, \varphi \in Q\left(A_{B}\right)$, we have

$$
\begin{aligned}
& u=v+w, v \in Q\left(A_{F}\right), w \in Q(B) \\
& \varphi=\vartheta+\zeta, \vartheta \in Q\left(A_{F}\right), \quad \zeta \in Q(B) .
\end{aligned}
$$

and since $v(R, \theta)=\vartheta(R, \theta)=0$ (which follows from the definition of $Q\left(A_{F}\right)$ ),

$$
\begin{gathered}
A^{*} w=0 \text { in } \Omega, \quad w(R)=u(R), \\
A^{*} \zeta=0 \text { in } \Omega, \quad \zeta(R)=\varphi(R) .
\end{gathered}
$$

Remark 4.1. Since $v(1, \theta)=0$ for any $v \in Q\left(A_{F}\right), Q\left(A_{F}\right)$ coincides with Brasche and Melgaard's form domain of $A_{F}$ in [4], and so $A_{F}$ is determined in their Theorem 4.5.

We now proceed as in the proof of Theorem 2.2. For $u=u_{F}+w \in$ $D\left(A_{B}\right)$ and $\varphi=\vartheta+\zeta \in Q\left(A_{B}\right)$

$$
\left(A_{B} u, \varphi\right)=\int_{\Omega}\left(A u_{F}\right) \bar{\varphi} d x
$$

and

$$
\left(A_{B} u, \varphi\right)=\int_{\Omega}\left(A u_{F}\right) \bar{\vartheta} d x+b[w, \zeta]
$$

Consequently

$$
b[w, \zeta]=\int_{\Omega}\left(A u_{F}\right) \bar{\zeta} d x
$$


If $\left\{\psi_{k}\right\}$ is an orthonormal basis of $Q(B)$, then we have with the same notation as in Sect. 3, that $u=u_{F}+w \in D\left(A_{B}\right)$ if and only if

$$
\forall k: \quad \sum_{j} b_{j k} w_{j}=\int_{\Omega}\left(A u_{F}\right) \overline{\psi_{k}} d x .
$$

The transformation

$$
W f(r)=r^{1 / 2} f(r), \quad f \in L^{2}(0,1 ; r d r)
$$

is a unitary operator from $L^{2}(0,1 ; r d r)$ onto $L^{2}(0,1)$, and as $\left\{e^{i m \theta} / \sqrt{2 \pi}\right\}_{m \in \mathbb{Z}}$ is an orthonormal basis of $L^{2}\left(\mathbb{S}^{1}\right)$ we have

$$
L^{2}(\Omega)=\bigoplus_{m \in \mathbb{Z}} W^{-1} L^{2}(0,1) \otimes \operatorname{Span}\left\{e^{i m \theta} / \sqrt{2 \pi}\right\} .
$$

In terms of this decomposition it follows that

$$
A=\bigoplus_{m \in \mathbb{Z}} W^{-1} T^{(m)} W \otimes 1,
$$

where $T^{(m)}$ is the closure in $L^{2}(0,1)$ of the operator defined on $C_{0}^{\infty}(0,1)$ by the Sturm-Liouville expression

$$
\tau^{m} y:=-y^{\prime \prime}+\left((m+\alpha)^{2}-1 / 4\right) r^{-2} y, m \in \mathbb{Z}, 0<\alpha<1,
$$

i.e., $T^{(m)}$ is the minimal operator in $L^{2}(0,1)$ generated by $\tau^{m}$. With $\nu=$ $m+\alpha$, the set $\left\{r^{1 / 2+\nu}, r^{1 / 2-\nu}\right\}$ is a fundamental system for $\tau^{m} u=0$. The expression $\tau^{m}$ is non-oscillatory. For $m=-1,0$, it is in the limit-circle case at 0 ; for all other values of $m$, it is in the limit-point case at 0 . It is regular at 1 for all values of $m$. Thus $T^{(m)}$ has deficiency indices $(2,2)$ for $m=-1,0$ and $(1,1)$ otherwise. We shall now apply results from [5] to determine the positive self-adjoint extensions of $T^{(m)}$ in $L^{2}(0,1)$ for all $m \in \mathbb{Z}$. Note that the singular point here is at the left endpoint of the interval $[0,1]$, i.e., it is the point 0 , unlike the analysis of [5], where it is at the right endpoint. If $S^{(m)}$ is one such extension, then

$$
\bigoplus_{m \in \mathbb{Z}} W^{-1} S^{(m)} W \otimes 1
$$

is a positive self-adjoint extension of $A$.

Remark 4.2. We note that it is unlikely that all positive self-extensions of $A$ are obtained in this way. This assertion is based on the situation for $A_{0}=$ $-\Delta+1$ from Example 3.8. As in (4.11),

$$
A_{0}=\bigoplus_{m \in \mathbb{Z}} W^{-1} T_{(m)} W \otimes 1
$$

where now, $T_{(m)}$ is the minimal operator generated by

$$
\tau_{m} y=-y^{\prime \prime}+\left(\left[m^{2}-1 / 4\right] r^{-2}+1\right) y, m \in \mathbb{Z} .
$$

At $0, \tau_{m}$ is non-oscillatory and in the limit-circle case for $m=0$ and is otherwise limit-point. As above for $A$, if $S_{(m)}$ is a positive self-adjoint extension of $T_{(m)}$ then 


$$
\bigoplus_{m \in \mathbb{Z}} W^{-1} S_{(m)} W \otimes 1
$$

is a positive self-adjoint extension of $A_{0}$. All such extensions have boundary conditions which depend on behaviour at 0 , in view of the presence of the extension $S_{(0)}$ of $T_{(0)}$ which has deficiency indices $(1,1)$. However in Remark 3.9 we saw that this is not so for the Krein-von Neumann extension of $A_{0}$ !

We shall proceed to determine the extensions $T^{(m)}$ in (4.11).

\subsection{The Case when $\tau^{m}$ is Limit Point at $0(m \neq-1,0)$}

Theorem 2.1 in [5] establishes a one-one correspondence between the positive self-adjoint extensions of $T^{(m)}$ in this case and the one-parameter family $\left\{T_{l}^{(m)}\right\}, 0 \leq l \leq \infty$ of restrictions of $\left(T^{(m)}\right)^{*}$ to the domains

$$
\mathcal{D}\left(T_{l}^{(m)}\right)=\left\{v: v \in \mathcal{D}\left(\left(T^{(m)}\right)^{*}\right), v^{\prime}(1)=\left[\psi^{\prime}(1)-l\|\psi\|^{2}\right] v(1)\right\} .
$$

Here $\psi$ is a real function in $L^{2}(0,1)$ which satisfies $\tau^{m} \psi=0$ and $\psi(1)=1$. We therefore have

$$
\psi(r)=r^{1 / 2+|\nu|}, \psi^{\prime}(1)=1 / 2+|\nu|,\|\psi\|^{2}=[2(1+|\nu|)]^{-1} .
$$

4.2. The Case when $\tau^{m}$ is Limit-Circle at $0(m=-1,0)$ and $\operatorname{dim} N_{B}=1$ From Theorem 2.2 in [5] and writing $T^{*}$ instead of $\left(T^{(m)}\right)^{*}$ for simplicity, it follows that the positive self-adjoint extensions of the operator $T^{(m)}$ which correspond to the pair $\left\{B, N_{B}\right\}$ in the KVB theory with $\operatorname{dim} N_{B}=1$ form a one-parameter family $T_{\beta}$ of restrictions of $T^{*}$ with domains

$$
\mathcal{D}\left(T_{\beta}\right):=\left\{v \in \mathcal{D}\left(T^{*}\right):\left[g^{2}\left\{\left(\frac{v}{g}\right)\left(\frac{\psi}{g}\right)^{\prime}-\left(\frac{v}{g}\right)^{\prime}\left(\frac{\psi}{g}\right)\right\}\right]_{0}^{1}=\beta v(1)\|\psi\|^{2}\right\},
$$

where $\psi$ is a real function in $N_{B}$ with $\psi(1)=1, g$ is the non-principal solution of $\tau^{m} u=0$ and $\beta \geq 0$. The non-principal solution is $r^{1 / 2-|\nu|}, \nu=m+\alpha$. The Wronskian $W$ is given by

$$
W(v, \psi)=g^{2}\left\{\left(\frac{v}{g}\right)\left(\frac{\psi}{g}\right)^{\prime}-\left(\frac{v}{g}\right)^{\prime}\left(\frac{\psi}{g}\right)\right\} .
$$

The limits at 0 of the first and the second terms in (4.17) exist separately. To see this, let

$$
\frac{g^{\prime \prime}(r)}{g(r)}=\left(\nu^{2}-1 / 4\right) r^{-2}=: q(r)
$$

Hence by the Jacobi identity [5, Equation (1.10)], for $v \in \mathcal{D}\left(T^{*}\right)$ we get

$$
-\frac{1}{g}\left[g^{2}\left(\frac{v}{g}\right)^{\prime}\right]^{\prime}=-v^{\prime \prime}+q v=T^{*} v \in L^{2}(0,1) .
$$


Thus, since $g \in L^{2}(0,1)$,

$$
-\left[g^{2}\left(\frac{v}{g}\right)^{\prime}\right]^{\prime}=g\left(T^{*} v\right) \in L^{1}(0,1)
$$

which implies that

$$
\lim _{r \rightarrow 0+}\left[g^{2}\left(\frac{v}{g}\right)^{\prime}\right](r) \quad \text { and } \quad \lim _{r \rightarrow 0+} g^{2}(r)\left(\frac{\psi}{g}\right)^{\prime}(r)
$$

both exist. From [9, Remark 3] (see also [5, (2.9)]), $\lim _{r \rightarrow 0+}(v / g)(r)$ exists, which confirms our assertion that the separate limits exist.

We shall now determine the boundary conditions satisfied by the selfadjoint extensions of $T^{(m)}$ in the two cases corresponding to $\nu=m+\alpha, m=$ $-1,0, \alpha \in(0,1)$.

4.2.1. The Case $\boldsymbol{m}=-\mathbf{1}, \boldsymbol{\nu}=-\mathbf{1}+\boldsymbol{\alpha} \in(-\mathbf{1 , 0})$. In this case, the non-principal solution is $g(r)=r^{1 / 2+\nu}$ and $\psi(r)=\gamma\left(C_{1} r^{1 / 2-\nu}+C_{2} r^{1 / 2+\nu}\right)$, where $\gamma=\left(C_{1}+C_{2}\right)^{-1}$ for $C_{1}, C_{2}$ are constants and $C_{1} \neq 0$. Thus,

$$
\left(\frac{\psi}{g}\right)(r)=\gamma\left(C_{1} r^{-2 \nu}+C_{2}\right), \quad g^{2}\left(\frac{\psi}{g}\right)^{\prime}(r)=-2 \gamma \nu C_{1},
$$

and so using (4.18)

$$
W(r)=\left(\frac{v(r)}{g(r)}\right)\left(-2 \gamma \nu C_{1}\right)-\left[g^{2}(r)\left(\frac{v}{g}\right)^{\prime}(r)\right]\left[\gamma\left(C_{1} r^{-2 \nu}+C_{2}\right)\right] .
$$

The value at $r=1$ is

$$
W(1)=-v^{\prime}(1)-v(1)\left[2 \gamma \nu C_{1}-1 / 2-\nu\right] .
$$

By (4.20) and since $\nu<0$, the limits at 0 of both terms in (4.21) exist and

$$
\begin{aligned}
\lim _{r \rightarrow 0+} W(r)= & -2 \gamma \nu C_{1} \lim _{r \rightarrow 0+} \frac{v(r)}{r^{1 / 2+\nu}}-\gamma C_{2} \lim _{r \rightarrow 0+} g^{2}(r)\left(\frac{v}{g}\right)^{\prime}(r) \\
= & -2 \gamma \nu C_{1} \lim _{r \rightarrow 0+} \frac{v(r)}{r^{1 / 2+\nu}} \\
& -\gamma C_{2} \lim _{r \rightarrow 0+}\left[r^{1 / 2+\nu} v^{\prime}(r)-(1 / 2+\nu) r^{-1 / 2+\nu} v(r)\right] .
\end{aligned}
$$

Thus the boundary condition for $A_{B}$ in this case is

$$
2 \gamma \nu C_{1}\left\{\lim _{r \rightarrow 0+} \frac{v(r)}{r^{1 / 2+\nu}}-v(1)\right\}+\left\{\gamma C_{2} \lim _{r \rightarrow 0+} f_{1}(r)-f_{1}(1)\right\}=\beta v(1)\|\psi\|^{2}
$$

where $f_{1}(r):=\left[r^{1 / 2+\nu} v^{\prime}(r)-(1 / 2+\nu) r^{-1 / 2+\nu} v(r)\right]$. 
4.2.2. The Case $\boldsymbol{m}=\mathbf{0}, \boldsymbol{\nu}=\boldsymbol{\alpha} \in(\mathbf{0}, \mathbf{1})$. This time, the non-principal solution is $g(r)=r^{1 / 2-\nu}$ and, with $\psi$ as above, we have

$$
\left(\frac{\psi}{g}\right)(r)=\gamma\left(C_{1}+C_{2} r^{2 \nu}\right), \quad g^{2}\left(\frac{\psi}{g}\right)^{\prime}(r)=2 \gamma \nu C_{2},
$$

giving

$$
W(r)=\left(\frac{v(r)}{g(r)}\right)\left(2 \gamma \nu C_{2}\right)-\left[g^{2}(r)\left(\frac{v}{g}\right)^{\prime}(r)\right]\left[\gamma\left(C_{1}+C_{2} r^{2 \nu}\right)\right] .
$$

Therefore

$$
W(1)=2 \gamma \nu C_{2} v(1)-\left[v^{\prime}(1)-(1 / 2-\nu) v(1)\right] .
$$

By (4.25) and since $\nu>0$, both limits at 0 in (4.26) exist and

$$
\begin{aligned}
\lim _{r \rightarrow 0+} W(r)= & 2 \gamma \nu C_{2} \lim _{r \rightarrow 0+} \frac{v(r)}{r^{1 / 2-\nu}}-\gamma C_{1} \lim _{r \rightarrow 0+} g^{2}(r)\left(\frac{v}{g}\right)^{\prime}(r) \\
= & 2 \gamma \nu C_{2} \lim _{r \rightarrow 0+} \frac{v(r)}{r^{1 / 2-\nu}} \\
& -\gamma C_{1} \lim _{r \rightarrow 0+}\left[r^{1 / 2-\nu} v^{\prime}(r)-(1 / 2-\nu) r^{-1 / 2-\nu} v(r)\right] .
\end{aligned}
$$

Thus the boundary condition in this case is

$$
2 \gamma \nu C_{2}\left\{\lim _{r \rightarrow 0+} \frac{v(r)}{r^{1 / 2-\nu}}-v(1)\right\}-\left\{\gamma C_{1} \lim _{r \rightarrow 0+} f_{2}(r)-f_{2}(1)\right\}=\beta v(1)\|\psi\|^{2},
$$

where $f_{2}(r):=\left[r^{1 / 2-\nu} v^{\prime}(r)-(1 / 2-\nu) r^{-1 / 2-\nu} v(r)\right]$.

4.3. The Case when $\tau^{m}$ is Limit-Circle at $0(m=-1,0)$ and $\operatorname{dim} N_{B}=2$ From [5, Theorem 2.2], we have

$$
\begin{aligned}
\mathcal{D}\left(A_{B}\right):=\{v & \in \mathcal{D}\left(A^{*}\right):\left[g^{2}\left\{\left(\frac{v}{g}\right)\left(\frac{\psi_{k}}{g}\right)^{\prime}-\left(\frac{v}{g}\right)^{\prime}\left(\frac{\psi_{k}}{g}\right)\right\}\right]_{0}^{1} \\
& \left.=\sum_{j=1}^{2} b_{j k} c_{j}, j=1,2\right\},
\end{aligned}
$$

where $B:=\left(b_{j k}\right)_{j, k=1,2}$ is a matrix of parameters which is non-negative, $\left\{\psi_{1}, \psi_{2}\right\}$ is a real orthonormal basis of $N_{B}$ and $c_{1}, c_{2}$ are determined by the values of $v / g$ at 0 and 1 :

$$
\frac{v}{g}(0)=\sum_{j=1}^{2} c_{j} \frac{\psi_{j}}{g}(0), \quad \frac{v}{g}(1)=\sum_{j=1}^{2} c_{j} \frac{\psi_{j}}{g}(1) .
$$

The main difference from the analysis of the previous section is that we now replace $\psi$ by an orthonormal basis $\left(\psi_{1}, \psi_{2}\right)$ obtained from the linearly independent basis elements

$$
r^{1 / 2-|\nu|} \quad \text { and } \quad r^{1 / 2+|\nu|}, \nu=m+\alpha .
$$


On using the Gram-Schmidt procedure, we obtain the orthogonal vectors

$$
r^{\frac{1}{2}-|\nu|} \text { and } r^{|\nu|+\frac{1}{2}}-(1-|\nu|) r^{\frac{1}{2}-|\nu|},
$$

and the orthonormal system

$$
\psi_{1}=\sqrt{2(1-|\nu|)} r^{\frac{1}{2}-|\nu|}, \quad \psi_{2}=\frac{\sqrt{2(1+|\nu|)}}{|\nu|}\left(r^{|\nu|+\frac{1}{2}}-(1-|\nu|) r^{\frac{1}{2}-|\nu|}\right) .
$$

The non-principal solution is $g(r)=r^{1 / 2-|\nu|}$ and we have

$$
\psi_{1} / g=\sqrt{2(1-|\nu|)}, \quad\left(\psi_{1} / g\right)^{\prime}=0
$$

and

$$
\psi_{2} / g=\frac{\sqrt{2(1+|\nu|)}}{|\nu|}\left(r^{2|\nu|}+|\nu|-1\right), \quad g^{2}\left(\psi_{2} / g\right)^{\prime}=2 \sqrt{2(|\nu|+1)} .
$$

Let

$$
W_{k}=g^{2}\left\{\left(\frac{v}{g}\right)\left(\frac{\psi_{k}}{g}\right)^{\prime}-\left(\frac{v}{g}\right)^{\prime}\left(\frac{\psi_{k}}{g}\right)\right\}, \quad k=1,2 .
$$

Then

$$
W_{1}(r)=-\sqrt{2(1-|\nu|)} g^{2}(r)\left(\frac{v}{g}\right)^{\prime}(r)
$$

and we set

$$
\Theta_{1}:=W_{1}(1)-W_{1}(0)=-\sqrt{2(1-|\nu|)}\left(\left(\frac{v}{g}\right)^{\prime}(1)-\lim _{r \rightarrow 0} g^{2}(r)\left(\frac{v}{g}\right)^{\prime}(r)\right) .
$$

Also

$$
W_{2}(r)=2 \sqrt{2(1+|\nu|)}\left(\frac{v}{g}\right)(r)-\frac{\sqrt{2(1+|\nu|)}}{|\nu|}\left(|\nu|-1+r^{2|\nu|}\right) g^{2}\left(\frac{v}{g}\right)^{\prime}(r)
$$

giving

$$
\begin{aligned}
\Theta_{2}:= & W_{2}(1)-W_{2}(0) \\
= & \sqrt{2(1+|\nu|)}\left[2 v(1)-\left(\frac{v}{g}\right)^{\prime}(1)\right] \\
& -\sqrt{2(1+|\nu|)} \lim _{r \rightarrow 0}\left\{2\left(\frac{v}{g}\right)(r)-\frac{1}{|\nu|}\left(|\nu|-1+r^{2|\nu|}\right) g^{2}\left(\frac{v}{g}\right)^{\prime}(r)\right\} .
\end{aligned}
$$

We also have

$$
V:=\left(\begin{array}{l}
v(1) / g(1) \\
v(0) / g(0)
\end{array}\right)=\left(\begin{array}{ll}
\sqrt{2(1-|\nu|)} & \sqrt{2(1+|\nu|)} \\
\sqrt{2(1-|\nu|)} & \sqrt{2(1+|\nu|)}(1-1 /|\nu|)
\end{array}\right)\left(\begin{array}{l}
c_{1} \\
c_{2}
\end{array}\right) .
$$

Setting $V=\Psi c$, where

$$
\Psi:=\left(\begin{array}{ll}
\sqrt{2(1-|\nu|)} & \sqrt{2(1+|\nu|)} \\
\sqrt{2(1-|\nu|)} & \sqrt{2(1+|\nu|)}(1-1 /|\nu|)
\end{array}\right)
$$


is invertible and has inverse

$$
\Psi^{-1}=\frac{|\nu|}{2 \sqrt{\left(1-|\nu|^{2}\right)}}\left(\begin{array}{ll}
\sqrt{2(1+|\nu|)}(1 /|\nu|-1) & \sqrt{2(1+|\nu|)} \\
\sqrt{2(1-|\nu|)} & -\sqrt{2(1-|\nu|)}
\end{array}\right) .
$$

The boundary condition in (4.30) is therefore

$$
\left(\begin{array}{c}
\Theta_{1} \\
\Theta_{2}
\end{array}\right)=B \Psi^{-1} V
$$

For the Krein-von Neumann extension $\left(T^{(m)}\right)_{K}$ of the one-dimensional operator, the boundary condition is determined by $\Theta_{1}=\Theta_{2}=0$ : Hence

$$
\lim _{r \rightarrow 0} g^{2}(r)\left(\frac{v}{g}\right)^{\prime}(r)=\left(\frac{v}{g}\right)^{\prime}
$$

and

$$
\lim _{r \rightarrow 0} 2\left(\frac{v}{g}\right)(r)=2 v(1)-\frac{1}{|\nu|}\left(\frac{v}{g}\right)^{\prime}(1) .
$$

Following Remark 2.1, the Friedrichs extension $\left(T^{(m)}\right)_{F}$ is obtained by $c_{1}=c_{2}=0$, so that the right hand side of (4.30) is finite. Thus, from (4.31), the boundary conditions are given by $(v / g)(0)=(v / g)(1)=0$, i.e.,

$$
\lim _{r \rightarrow 0} \frac{v(r)}{r^{1 / 2-|\nu|}}=v(1)=0
$$

Open Access. This article is distributed under the terms of the Creative Commons Attribution 4.0 International License (http://creativecommons.org/licenses/by/4. 0/), which permits unrestricted use, distribution, and reproduction in any medium, provided you give appropriate credit to the original author(s) and the source, provide a link to the Creative Commons license, and indicate if changes were made.

Publisher's Note Springer Nature remains neutral with regard to jurisdictional claims in published maps and institutional affiliations.

\section{References}

[1] Abels, H., Grubb, G., Wood, I.G.: Extension theory and Krel̆n-type resolvent formulas for nonsmooth boundary value problems. J. Funct. Anal. 266(7), 4037-4100 (2014)

[2] Alonso, A., Simon, B.: The Birman-Kreı̆n-Vishik theory of selfadjoint extensions of semibounded operators. J. Oper. Theory 4(2), 251-270 (1980)

[3] Birman, M.Š.: On the theory of self-adjoint extensions of positive definite operators. Mat. Sb. N.S. 38(80), 431-450 (1956)

[4] Brasche, J.F., Melgaard, M.: The Friedrichs extension of the Aharonov-Bohm Hamiltonian on a disc. Integral Equ. Oper. Theory 2(3), 419-436 (2005)

[5] Brown, B.M., Evans, W.D.: Selfadjoint and $m$ sectorial extensions of SturmLiouville operators. Integral Equ. Oper. Theory 85(2), 151-166 (2016)

[6] Brown, B.M., Grubb, G., Wood, I.G.: M-functions for closed extensions of adjoint pairs of operators, with applications to elliptic boundary problems. Math. Nachr. 282, 314-347 (2009) 
[7] Edmunds, D.E., Evans, W.D.: Spectral Theory and Differential Operators. Clarendon Press, Oxford (1989)

[8] Grubb, G.: A characterization of the non-local boundary value problems associated with an elliptic operator. Ann. Scuola Norm. Sup. Pisa 3(22), 425-513 (1968)

[9] Kalf, H.: A characterization of the Friedrichs extension of Sturm-Liouville operators. J. Lond. Math. Soc. (2) 17(3), 511-521 (1978)

[10] Kochubei, A.N.: Extensions of symmetric operators and symmetric binary relations. Math. Notes 17(1), 25-28 (1975)

[11] Kreı̆n, M.: The theory of self-adjoint extensions of semi-bounded Hermitian transformations and its applications. I. Rec. Math. [Mat. Sb.] N.S. 20(62), 431-495 (1947)

[12] Krel̆n, M.: The theory of self-adjoint extensions of semi-bounded Hermitian transformations and its applications. II. Mat. Sb. N.S. 21(63), 365-404 (1947)

[13] Rellich, F.: Halbbeschränkte gewöhnliche Differentialoperatoren zweiter Ordnung. Math. Ann. 122, 343-368 (1951)

[14] Rosenberger, R.: A new characterization of the Friedrichs extension of semibounded Sturm-Liouville operators. J. Lond. Math. Soc. (2) 31(3), 501-510 (1985)

[15] Vishik, M.: On general boundary conditions for elliptic differential operators. Trudy Moskov. Mat. Obsc. (Russian). (English translation In: Amer. Math. Soc. Transl. 24, 107-172), 187-246 (1952)

B. M. Brown

Cardiff School of Computer Science and Informatics

Cardiff University

Queen's Buildings, 5 The Parade, Roath

Cardiff CF24 3AA

UK

e-mail: malcolm@cs.cf.ac.uk

W. D. Evans

School of Mathematics

Cardiff University

Senghennydd Road

Cardiff CF24 4AG

UK

e-mail: evanswd@cf .ac.uk

I. G. Wood $(\bowtie)$

School of Mathematics, Statistics and Actuarial Science

University of Kent

Sibson Building

Canterbury CT2 7FS

UK

e-mail: i.wood@kent.ac.uk

Received: November 28, 2018.

Revised: August 5, 2019. 